\title{
Paciente adulto con diabetes mellitus tipo 1: múltiples factores para lograr un control adecuado. Reporte de caso
}

Juan Carlos Lizarzaburu Robles*1,2,a; Juliana María Vera Ortiz 2,b; Sonia Gisella Chia Gonzales 3,a

\section{RESUMEN}

La diabetes mellitus tipo 1 (DM1) es una de las enfermedades crónicas más comunes de la infancia; sin embargo, un gran número de pacientes adultos viven con esta condición. La terapia con insulina es el pilar del tratamiento. Otros aspectos importantes son fomentar un estilo de vida saludable y lograr la adherencia a la medicación. Se presenta el caso de una mujer de 29 años con diagnóstico de diabetes mellitus tipo 1 hace 15 años. Describimos el manejo y evolución de los últimos 5 años y destacamos la importancia de las nuevas tecnologías, como el monitoreo continuo de glucosa, además de factores como la adherencia al tratamiento y el soporte familiar, los cuales fueron particularmente importantes para el control de la enfermedad de la paciente.

Palabras clave: Diabetes mellitus tipo 1; Cumplimiento y adherencia al tratamiento; Automonitorización de la glucosa sanguínea (Fuente: DeCS BIREME).

\section{Adult patient with type 1 diabetes mellitus: multiple factors to achieve an adequate control}

\section{ABSTRACT}

Type 1 diabetes mellitus (T1DM) is one of the most common chronic childhood diseases. However, a large number of adult patients live with this condition. Insulin therapy is the cornerstone of diabetes treatment. Other important aspects are the promotion of a healthy lifestyle and the adhesion to medications. This is the case of a 29-year-old woman with a fifteenyear diagnosis of T1DM. Medical treatment and evolution over the last five years are described. The importance of new technologies such as continuous glucose monitoring and other factors such as adherence to treatment and family support were particularly important for the patient's adequate disease control.

Keywords: Diabetes mellitus, type 1; Treatment adherence and compliance; Blood glucose self-monitoring (Source: MeSH NLM).

\footnotetext{
1 Hospital Central de la Fuerza Aérea Peruana. Lima, Perú.

2 Asociación para la prevención, educación e investigación en diabetes- APREDIAB. Lima, Perú.

3 Hospital Nacional Cayetano Heredia. Lima, Perú.

a Médico, especialista en Endocrinología.

b Médico, especialista en Endocrinología Pediátrica.

*Autor corresponsal.
} 


\section{INTRODUCCIÓN}

El tratamiento y control de la diabetes mellitus tipo 1 (DM1) es complejo y demandante. Durante la última década, el conocimiento de la patogénesis y de la historia natural de la enfermedad se ha ampliado considerablemente. No obstante, aún no existen medios para prevenir o curar esta enfermedad ${ }^{(1,2)}$.

La prevalencia de diabetes se está incrementando. Aunque gran parte de este fenómeno es el resultado de la epidemia de diabetes tipo 2, datos epidemiológicos sugieren que la incidencia de DM1 está aumentando en un 2-5 \% por año en todo el mundo ${ }^{(3,4)}$. Las razones de este incremento no son claras, aunque una asociación entre la genética y factores ambientales pudiera estar involucrada ${ }^{(5)}$. En el Perú, se reporta una de las incidencias más bajas de DM1 (<1 caso /100 000 habitantes /año en menores de 15 años), en las localidades de Lima, Cusco y San Martín ${ }^{(6,7)}$. Sin embargo, esta cifra pudiera estar subestimada en la actualidad.

Un adecuado control glicémico ha demostrado reducir el riesgo de desarrollar complicaciones crónicas y la mortalidad cardiovascular en pacientes con DM1 ${ }^{(8)}$. La prueba sugerida para el seguimiento de la enfermedad es la hemoglobina glicosilada (HbA1c) y el valor recomendado por la American Diabetes Association (ADA) es $\leq 7 \%$ en pacientes adultos ${ }^{(9)}$. En Latinoamérica, se reporta un alto porcentaje de pacientes con DM1 que no logran un control glicémico adecuado y mantienen el valor de HbA1c por encima de lo recomendado ${ }^{(10)}$. Actualmente, el monitoreo continuo de glucosa (MCG) se han convertido en una importante herramienta para el control y seguimiento del paciente, y le permite tener un registro continuo de los valores de glucosa en sangre ${ }^{(11-13)}$. El control nutricional y actividad física también son importantes, aunque con menor nivel de evidencia ${ }^{(14)}$.

El soporte familiar o la ansiedad ante un estricto control son, asimismo parte de la perspectiva del tratamiento en DM1; sin embargo, no son expuestos frecuentemente en la literatura ${ }^{(2,3)}$. Nuestro objetivo es describir y revisar los múltiples factores que influyen en el adecuado control de un paciente con DM1 y resaltar el uso de nuevos dispositivos de control, así como la importancia del soporte familiar y una buena adherencia al tratamiento.

\section{CASO CLÍNICO}

Paciente mujer de 29 años con diagnóstico de DM1 a los 14 años. Desde el inicio de la enfermedad recibió tratamiento con insulina en diversos esquemas y ha sido evaluada en diferentes centros médicos durante los primeros años de seguimiento. Los controles de glicemia y $\mathrm{HbA} 1 \mathrm{c}$ no fueron adecuados durante ese periodo. A pesar de ello, no fue hospitalizada por complicaciones agudas. Se describe el seguimiento por la especialidad de endocrinología en tres periodos diferentes durante los últimos cinco años de tratamiento.

En el control inicial (2013), la paciente presentaba una glicemia en ayunas (GA) de $184 \mathrm{mg} / \mathrm{dl}$ y una HbA1c en $10,8 \%$ (en la tabla 1 se muestran otros valores); además, refería fatiga y urgencia para la micción. Peso: $79 \mathrm{~kg}$, talla: 1,67 m e índice de masa corporal (IMC): 28,3. El MCG inicial evidencia hiperglicemias persistentes y un tiempo de "rango objetivo" (R) de 17 \% (Figura 1a). Con esta información el régimen de insulina se modificó, y se indicó tratamiento con insulinas análogas (glargina basal y asparta con las comidas). La paciente fue instruida para que realice automonitoreo de la glucosa sanguínea y se le sugirió realizar actividad física regular y asesoría nutricional. La paciente presentó buena adherencia al tratamiento médico, pero no al control nutricional ni actividad física.

Tabla 1. Exámenes auxiliares registrados por año del control descrito

\begin{tabular}{|lccc}
\hline & $\begin{array}{c}\text { Control } \\
\text { inicial } \\
\text { Exámenes } \\
\text { auxiliares }\end{array}$ & $\begin{array}{c}\text { 3. }^{\text {er }} \text { año } \\
\text { de control } \\
(2013)\end{array}$ & $\begin{array}{c}5 .^{\circ} \text { año } \\
\text { de control } \\
(2018)\end{array}$ \\
\hline Glucosa basal (mg/dl) & 184 & 107 & 124,9 \\
\hline HbA1c * (\%) & 10,8 & 7,1 & 7,7 \\
\hline Colesterol total (mg/dl) & 186,4 & 187,9 & 158,3 \\
\hline Triglicéridos (mg/dl) & 199 & 135,2 & 146,9 \\
\hline Colesterol HDL (mg/dl) & 53,4 & 48.9 & 3,8 \\
\hline Colesterol LDL (mg/dl) & 93,2 & 112 & 90,1 \\
\hline Urea (mg/dl) & 25,8 & 27 & 24,2 \\
\hline Creatinina (mg/dl) & 0,9 & 0,8 & 0,7 \\
\hline Microalbuminuria (ug/min) & 4,5 & 8,1 & 7,1 \\
\hline TGO (mg/dl) & 25,3 & 22.7 & 18 \\
\hline TGP (mg/dl) & 33,6 & 30 & 31,6 \\
\hline Hb-Hto (gr-\%) & $13,6-40 \%$ & $13,7-4,1 \%$ & $13,6-40 \%$ \\
\hline Péptido-C (nmol/L) & 0,4 & - & - \\
\hline TSH (u IU/ml) & 1,3 & 1,1 & 0,9 \\
\hline
\end{tabular}

*HbA1c: Hemoglobina glicosilada A1c, TGO: Transaminasa glutámica oxalacetica

TGP: Transaminasa glutámico-pirúvica, TSH: Hormona estimulante de la tiroides 
En el tercer año de seguimiento, la paciente mostraba mejoría en el control glucémico (GA en $107 \mathrm{mg} / \mathrm{dl}$ y HbA1c del 7,1\%). Sin embargo, aumentó de peso (82 kg) y el IMC alcanzó un valor de 29. Se reportaron síntomas asociados a hipoglicemia leve y esporádica durante este periodo (somnolencia y apetito incrementado). El control de MCG muestra menores excursiones de hiperglicemia y un tiempo de R de $22 \%$, sin evidencia hipoglicemia (Figura 1b). Las dosis de insulina se ajustaron y se indicó control nutricional. La paciente inicio y logró la adherencia a un programa de actividad física regular (danza o baile tradicional) y mantuvo el control médico regular.

En el quinto año de seguimiento, la paciente presentó una glucosa en ayunas de $124 \mathrm{mg} / \mathrm{dl}$ y HbA1c de 7,7 \%. No refirió episodios asociados a hipoglicemia sintomática y se registró una baja de peso $(80 \mathrm{~kg})$ respecto al control previo (IMC: 28,7$)$. Se observó un mejor control en el MCG con un tiempo de $\mathrm{R}$ de 58 \% (Figura 1c). La evolución clínica fue favorable y logra mantener su rutina de actividad física, pero no un régimen nutricional adecuado.

Durante los cinco años de seguimiento descritos la paciente no presentó complicaciones agudas que requieran su hospitalización, como cetoacidosis diabética o hipoglicemia severa. La función renal, el examen oftalmológico y de miembros inferiores no presentaron alteraciones asociadas con complicaciones crónicas.

a. MCG Inicial (2013)

b. MCG Tercer año (2016)
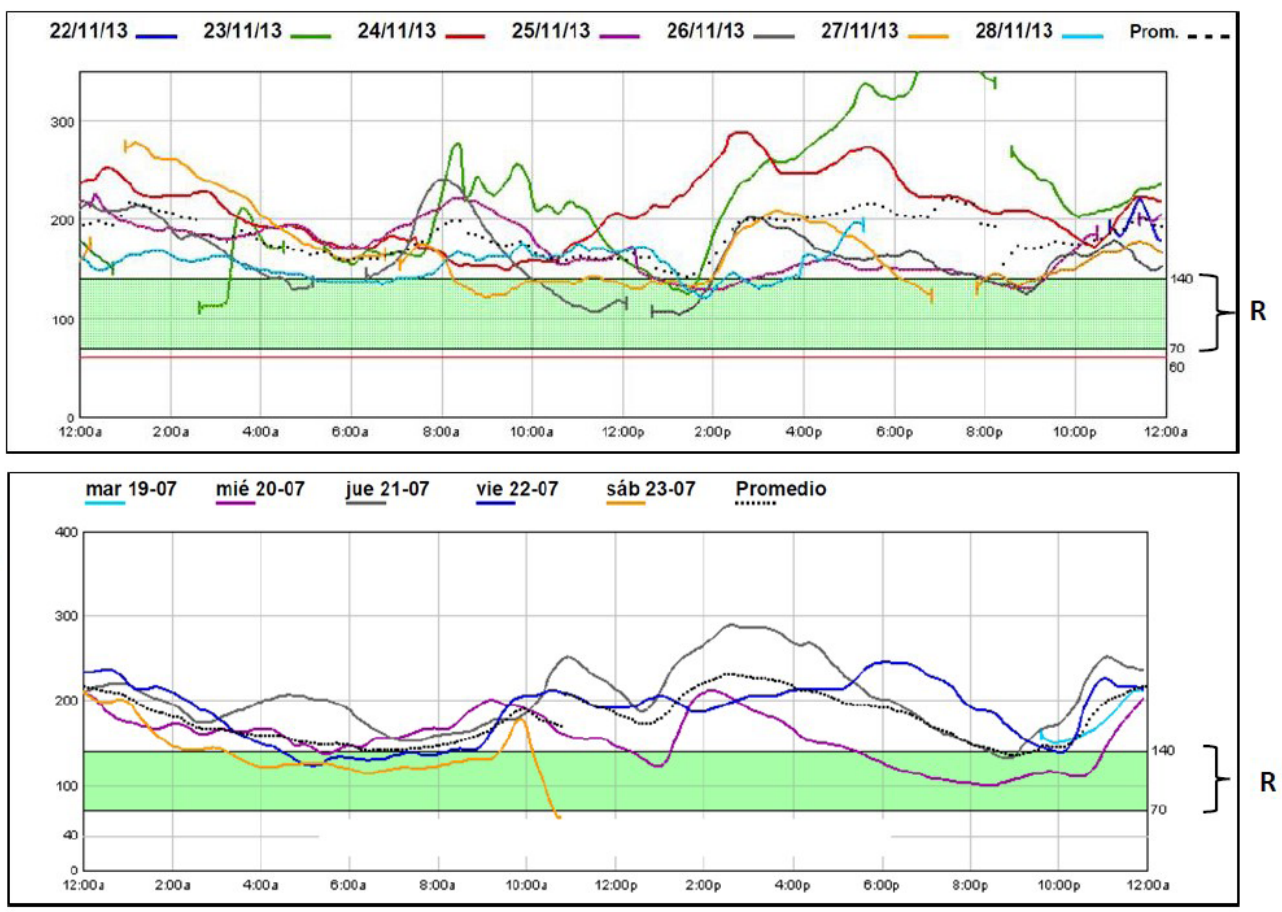

C. MCG Quinto año (2018)

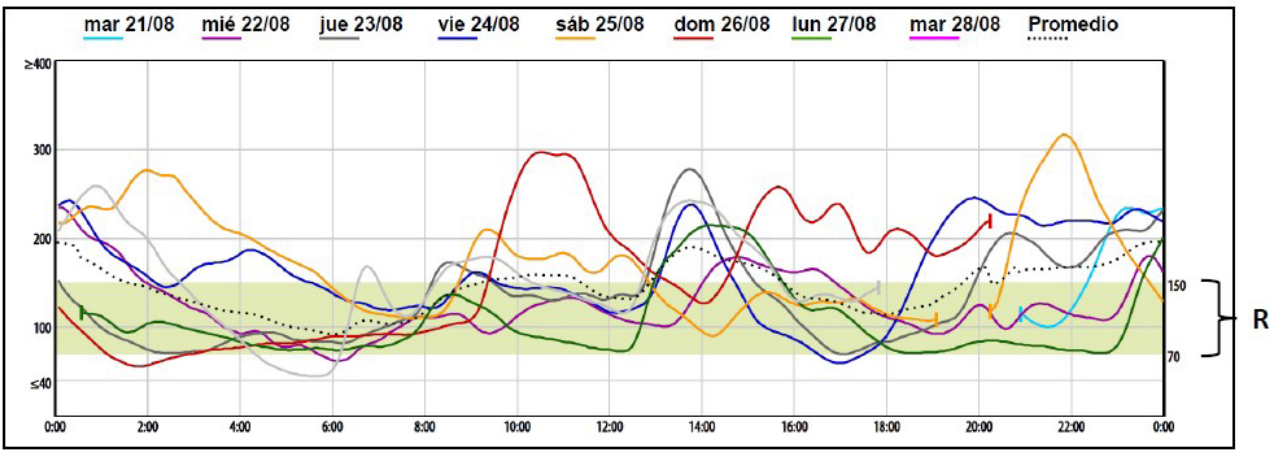

Figura 1. Gráficas de monitoreo continuo de glucosa en los años de seguimiento descritos MCG: Monitoreo continuo de glucosa R: Rango objetivo 


\section{DISCUSIÓN}

La DM1 es una enfermedad metabólica que resulta un desafío para lograr un adecuado control. Puede ser diagnosticada a cualquier edad, pero los picos en la presentación ocurren entre los 5 a 7 años y cerca de la pubertad (1). Hoy , y como resultado de mejores tratamientos para de la enfermedad, existe un incremento del número de pacientes adultos con esta condición ${ }^{(4,15)}$. Es interesante mencionar que mientras la mayoría de los trastornos autoinmunes afectan a las mujeres, la diabetes tipo 1 es un poco más común en varones ${ }^{(1)}$.

Actualmente, la terapia con insulina es el estándar para el tratamiento de la DM1. Antes de su descubrimiento en $1922,50 \%$ de los pacientes fallecían dentro de los primeros 20 meses de diagnóstico, y menos del 10 \% sobrevivían a los cinco años ${ }^{(16)}$. Las limitaciones derivadas del costo, el riesgo de hipoglicemia y algunos mitos sobre la enfermedad, pueden ser una barrera para su uso. En el caso de nuestra paciente, se mantuvo una adherencia continua al empleo del medicamento.

\section{Control glicémico y metas:}

Hemoglobina glicosilada. Pocos pacientes logran un valor óptimo de HbA1c. El valor objetivo recomendado de hemoglobina glicosilada $\mathrm{A} 1 \mathrm{C}$ es $\leq 7 \%$ en adultos ${ }^{(9)} \mathrm{y} \leq$ $7,5 \%$ en niños con DM1, debido al riesgo de hipoglicemia y las dificultades para lograr un valor más bajo ${ }^{(17,18)}$. Sin embargo, este valor debe ser individualizado. Nuestra paciente logró un valor óptimo y sostenido de HbA1c en el tiempo. La importancia del control de $\mathrm{HbA} 1 \mathrm{c}$ radica en la evidencia del menor riesgo de complicaciones microvasculares y desenlaces fatales por eventos coronarios (8). Recientemente, un estudio poblacional en niños y adultos con DM1 mostró que el riesgo de complicaciones graves como retinopatía y nefropatía aparece, principalmente, con niveles de $\mathrm{HbA} 1 \mathrm{c}>8,6 \%$, pero para valores de $\mathrm{HbA} 1 \mathrm{c}>$ $7,0 \%$ ya se presentaban complicaciones leves ${ }^{(17)}$.

Glicemia de ayunas y control de lípidos. En el seguimiento de la paciente, los valores de glicemia de ayunas registraron una mejoría. Sin embargo, un control de glucosa venosa obtenido en ayunas no reflejaría un parámetro adecuado de seguimiento. No existen referencias actuales sobre su utilidad. Respecto al control de lípidos (colesterol total, colesterol LDL, colesterol HDL y triglicéridos), la ADA, sugiere solicitarlo en el momento del diagnóstico y se justifica repetirlo con una frecuencia relativa en pacientes

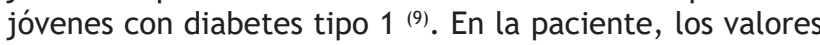
de lípidos estuvieron en rangos adecuados durante los controles, por lo que no se justifica la intervención farmacológica.

Automonitoreo y monitoreo continuo de glucosa. En pacientes con DM1 está recomendado llevar un automonitoreo de la glucosa sanguínea con glucómetro varias veces al día (hasta 6-10 veces / día) que incluyen controles antes de las comidas, a la hora de acostarse y en situaciones específicas como el ejercicio o si el paciente conduce un vehículo ${ }^{(9,18)}$. En el caso de la paciente, el automonitoreo de la glucosa sanguínea contribuyó a mejorar el control glicémico. El uso del glucómetro puede ayudar a guiar el tratamiento y / o el autocontrol para pacientes con uso de múltiples dosis de insulina ${ }^{(9,11,18)}$.

El monitoreo continuo de glucosa (MCG) se ha convertido en la actualidad en una importante herramienta para lograr el buen control glicémico de los pacientes ${ }^{(11,13,18)}$. En adultos con DM1 se ha demostrado un mejor control glucémico en aquellos que usan un MCG ${ }^{(19)}$. Un metaanálisis de 25 estudios concluyó que el uso de este dispositivo da como resultado una reducción significativa y sostenida de $\mathrm{HbA1c}$ para adultos y niños con diabetes tipo $1^{(20)}$. A diferencia de la HbA1c, el MCG nos muestra las fluctuaciones de glicemia (hiper e hipoglicemia), además del periodo (\%) del tiempo de $\mathrm{R}$ de glucosa que presenta el paciente ${ }^{(11,13)}$. Con esta información (Figura 1) decidimos mejores cambios en las dosis y horarios de administración de insulina. El dispositivo utilizado fue un iPro2, el cual es un sistema MCG profesional o "ciego" compuesto por un sensor que registra los niveles de glucosa durante 24 horas por 7 días. Los datos del MCG se obtienen cuando el dispositivo es revisado de manera retrospectiva por el médico ${ }^{(11,12)}$.

Estilos de vida: nutrición y actividad física. El plan nutricional no fue seguido de manera regular por la paciente. Aumentó de peso hacia el tercer año de control. Refería variaciones constantes en el horario y tipo de comidas asociados al ritmo de estudio y trabajo. Está descrito que las estrategias dietéticas complementan la terapia farmacológica. En una revisión sistemática de dietas bajas en carbohidratos, se demostró una reducción significativa de HbA1c en paciente con DM1 (21). Otro elemento importante para el control de la DM1 es la actividad física. En Inglaterra, un metaanálisis de 23 estudios en DM1 reveló que la actividad física reduce la HbA1c y colesterol total ${ }^{(22)}$. La paciente mejoro su actividad física, luego de iniciar y mostrar adherencia a una rutina de baile tradicional, lo que pudo influir en la autoestima de la paciente y en el adecuado control glicémico.

\section{Otros factores asociados al control glicémico}

Soporte familiar: Se ha descrito que la participación continua de los padres en el control de la DM1 es importante en aspectos como la adherencia a la medicación o el monitoreo de glucosa. Una etapa fundamental es la transición de la niñez a la adolescencia, observándose mejores resultados cuando esta participación ocurre de una manera cálida y colaborativa ${ }^{(2,23)}$. En el caso de la paciente, había un compromiso familiar, se mantuvo una 
comunicación permanente y el estilo de vida del hogar se adaptó para apoyarla. El desarrollo de una actividad cultural (danza), aceptada por los padres, mejoró la motivación de la paciente.

Ansiedad y estrés: El tratamiento de la DM1 debería ser integral y requiere profundizar en todos los aspectos de la enfermedad (clínicos y psicológicos) con equipos multidisciplinarios que incluyen profesionales como trabajadores sociales y psicólogos ${ }^{(24)}$. No es raro que muchos adolescentes, sientan ira, miedo, infelicidad o, incluso, crisis de angustia por el rechazó a la enfermedad ${ }^{(25)}$. En Latinoamérica, los jóvenes con DM1 han reportado altos niveles de estrés, debido a los serios y complejos desafíos psicológicos, los que pudieran provocar síntomas depresivos, mala adherencia a la insulinoterapia $\mathrm{y}$, en consecuencia, un mal control metabólico ${ }^{(24)}$. En el caso de la paciente, el soporte familiar se consideró importante para mitigar la ansiedad y el estrés. No se reportó negación de enfermedad. Sin embargo, pudieron presentarse estados de angustia o miedo.

Adherencia al tratamiento: Existen competencias neuropsicológicas de los pacientes y familiares, especialmente, en el dominio del "funcionamiento ejecutivo", que es la capacidad de autocontrolarse, planificar, resolver problemas y establecer prioridades. Un estudio encontró una interacción significativa entre el dominio de funcionamiento ejecutivo infantil y parental, y la asociación con la adherencia al tratamiento y el control glucémico en DM1, en este punto se debería trabajar conjuntamente para obtener mayor cumplimiento ${ }^{(26)}$. La paciente presentó una buena adherencia al tratamiento médico, mas no al régimen nutricional. La tecnología, a través del autocontrol apoyado por teléfonos inteligentes que ofrecen registros de glicemias, seguimiento de actividad física o recomendación de dosis de insulina (27), podría contribuir a una mayor adherencia, aunque aún no existe suficiente información.

El adecuado control del paciente adulto con DM1 depende de múltiples factores. Los avances de la tecnología ofrecen mejores opciones para el tratamiento como insulinas análogas o monitores continuos de glucosa. Sin embargo, también es necesario considerar aspectos como el soporte familiar o la adherencia al tratamiento para alcanzar las metas de control.

Contribuciones de los autores: Juan Carlos Lizarzaburu Robles contribuyó con la descripción del caso, seguimiento del paciente, revisión de los exámenes auxiliares y elaboración del manuscrito. Juliana Vera participó en la descripción del caso y revisión del manuscrito. Sonia Chía contribuyó en la revisión bibliográfica y revisión del manuscrito.
Conflicto de interés: Los autores declaran no tener conflictos de interés.

Fuentes de financiamiento: El artículo fue financiado por los autores.

\section{REFERENCIAS BIBLIOGRÁFICAS}

1. Atkinson MA, Eisenbarth GS, Michels AW. Type 1 diabetes. Lancet. 2014; 383(9911): 69-82.

2. Jaser SS. Family interaction in pediatric diabetes. Curr Diab Rep. 2011; 11(6): 480-5.

3. Freeborn D, Dyches T, Roper SO. Lessons Learned from a life with type 1 diabetes: adult perspectives. Diabetes Spectr. 2017; 30(3): 188-94.

4. Dhaliwal R, Weinstock RS. Management of type 1 diabetes in older adults. Diabetes Spectr. 2014; 27(1): 9-20.

5. Cho NH, Shaw JE, Karuranga S, Huang Y, da Roche Fernandes JD, Ohlrogge AW, et al. IDF diabetes atlas: global estimates of diabetes prevalence for 2017 and projections for 2045. Diabetes Res Clin Pract. 2018; 138: 271-81.

6. Karvonen M, Viik-Kajander M, Moltchanova E, Libman I, Laporte R, Tuomilehto J. Incidence of childhood type 1 diabetes worldwide. Diabetes Mondiale (DiaMond) Project Group. 2000; 23(10): 1516-26.

7. Seclén S, Rojas MI. Diabetes Mellitus Tipo 1 en población infantil peruana. Diagnóstico. 2002; 41(2): 54-62.

8. Lind $M$, Svensson A-M, Rosengren A. Glycemic control and excess mortality in type 1 diabetes. N Engl J Med. 2015; 372(9): 880-1.

9. American Diabetes Association. Standards of Medical Care in Diabetes-2018. Abridged for Primary Care Providers. Clin Diabetes. 2018; 36(1): 14-37.

10. Palmezano-Díaz JM, Figueroa-Pineda CL, Rodríguez R, Plazas-Rey L, Corredor-Guzmán K, Pradilla-Suárez LP, et al. Características clínicas y sociodemográficas de pacientes con diabetes tipo 1 en un Hospital Universitario de Colombia. Med Int Méx. 2018; 34(1): 46-56.

11. Bzowyckyj A. Updates and advances in technology for diabetes selfmanagement. Pharmacy Today. 2019; 25(5): 43-58.

12. Foster NC, Beck RW, Miller KM, Clements MA, Rickels MR, DiMeglio LA, et al. State of type 1 diabetes management and outcomes from the T1D exchange in 2016-2018. Diabetes Technol Ther. 2019; 21(2): 66-72.

13. Hortensius J, Kars MC, Wierenga WS, Kleefstra N, Bilo HJ, van der Bijl JJ. Perspectives of patients with type 1 or insulin-treated type 2 diabetes on self-monitoring of blood glucose: a qualitative study. BMC Public Health. 2012; 12(1): 167.

14. NICE Guideline 18 [NG18]. Diabetes (type 1 and type 2) in children and young people: diagnosis and management. Methods, evidence and recommendations. 2016.

15. Vehik K, Dabelea D. The changing epidemiology of type 1 diabetes: why is it going through the roof?. Diabetes Metab Res Rev. 2011; 27(1): 3-13.

16. Katz M, Laffel L. Mortality in type 1 diabetes in the current era: two steps forward, one step backward. JAMA. 2015; 313(1): 35-6.

17. Lind M, Pivodic A, Svensson A-M, Ólafsdóttir AF, Wedel H, Ludvigsson J. HbA 1c level as a risk factor for retinopathy and nephropathy in children and adults with type 1 diabetes: Swedish population based cohort study. BMJ. 2019; 366: 14894.

18. Chiang JL, Maahs DM, Garvey KC, Hood KK, Laffel LM, Weinzimer $\mathrm{SA}$, et al. Type 1 diabetes in children and adolescents: a position statement by the American Diabetes Association. Diabetes Care. 2018; 41(9): 2026-44. 
19. Juvenile Diabetes Research Foundation Continuous Glucose Monitoring Study Group, Tamborlane WV, Beck RW, Bode BW, Buckingham B, Chase HP, et al. Continuous glucose monitoring and intensive treatment of type 1 diabetes. N Engl J Med. 2008; 359(14): $1464-76$

20. Evans M, Welsh Z, Ells S, Seibold A. The impact of flash glucose monitoring on glycaemic control as measured by HbA1c: a metaanalysis of clinical trials and real-world observational studies. Diabetes Ther. 2020; 11(1): 83-95.

21. Turton JL, Raab R, Rooney KB. Low-carbohydrate diets for type 1 diabetes mellitus: a systematic review. PLoS One. 2018; 13(3): e0194987.

22. Quirk H, Blake H, Tennyson R, Randell TL, Glazebrook C. Systematic review or meta-analysis physical activity interventions in children and young people with type 1 diabetes mellitus: a systematic review with meta-analysis. Diabet Med. 2014; 31(1): 1163-73.

23. Goethals ER, Oris L, Soenens B, Berg CA, Prikken S, Broeck NV, et al. Parenting and treatment adherence in type 1 diabetes throughout adolescence and emerging adulthood. J Pediatr Psychol. 2017; 42(9): 922-32.

24. Henríquez-Tejo R, Cartes-Velásquez R. Psychosocial impact of type 1 diabetes mellitus in children, adolescents and their families. Literature review. Rev Chil Pediatr. 2018; 89(3): 391-8.

25. Lesley Lowes, Eddy D, Channon S, McNamara R, Robling M, Gregory JW, et al. The experience of living with type 1 diabetes and attending clinic from the perception of children, adolescents and carers: analysis of qualitative data from the DEPICTED study. J Pediatr Nurs. 2015; 30(1): 54-62.

26. Goethals ER, de Wit M, Van Broeck N, Lemiere J, Van Liefferinge $\mathrm{D}$, Bohler $\mathrm{S}$, et al. Child and parental executive functioning in type 1 diabetes: Their unique and interactive role toward treatment adherence and glycemic control. Pediatr Diabetes. 2018; 19(3): 520-6.

27. Beck RW, Bergenstal RM, Laffel LM, Pickup JC. Advances in technology for management of type 1 diabetes. Lancet. 2019; 394(10205): 1265-73.
Correspondencia:

Juan Carlos Lizarzaburu Robles

Dirección: Calle San Antonio 138, La Molina. Lima, Perú. Teléfono: 511- 997419121

Correo electrónico: juancarlosliro@yahoo.com

Recibido: 28 de enero de 2020

Evaluado: 24 de agosto de 2020

Aprobado: 10 de setiembre de 2020

(c) La revista. Publicado por Universidad de San Martín de Porres, Perú. (cc) BY Licencia de Creative Commons Artículo en acceso abierto bajo términos de Licencia Creative Commons Atribución 4.0 Internacional. (http://creativecommons.org/licenses/by/4.0/)

ORCID iDs

Juan Carlos Lizarzaburu Robles 10 https: / / orcid.org/0000-0003-4542-8679

Juliana María Vera Ortiz C https://orcid.org/0000-0002-2266-433X

Sonia Gisella Chia Gonzales $\quad$ https://orcid.org/0000-0002-4387-8898 